superconductor. Therefore, Majorana fermions should exist on the edge of $\mathrm{\alpha}-\mathrm{RuCl}_{3}$.

The direct detection of Majorana fermions in condensed-matter systems was never going to be easy. Such particles must be electrically neutral and therefore cannot participate in electrical transport (although they can mediate such transport in superconductors ${ }^{8,9}$ ). However, although Majorana fermions are unable to conduct current, they can conduct heat.

Electrons can conduct both electricity and heat. As a result, metals - which contain many free electrons - are typically good heat conductors. This idea is formalized by the Wiedemann-Franz law, which states that electrical conductivity is directly proportional to thermal conductivity divided by temperature. Although the identification of this relationship is often lauded as one of the early successes of solid-state theory, the proportionality constant is not universal for ordinary metals: scattering processes, which limit both electrical and thermal conductivity, affect these properties differently in different metals.

However, if the motion of particles in a material is ballistic (if there is effectively no scattering), both electrical and thermal conductivity are quantized and proportional to the number of propagating modes (conduction channels). Each electron mode contributes a unit of thermal conductance, and, crucially, each Majorana mode contributes only half a unit. Both Banerjee et al. and Kasahara et al. observed this fraction of thermal conductance on the edges of their condensed-matter systems.

The existence of Majorana edge modes in a condensed-matter system is a strong indicator that the topological order of the system is non-Abelian - which means, for example, that a collection of the system's excitations has a huge number of quantum states with the same energy. The non-Abelian nature of the quantum Hall state studied by Banerjee et al. has long been expected (albeit not confirmed beyond reasonable doubt). However, Kasahara and colleagues' findings provide the first experimental evidence of a non-Abelian spin liquid. Although more work is needed to confirm the exact nature of this state, the discovery of such an unconventional phase of matter is truly exciting.

Banerjee and colleagues used their measurements to try to discriminate between different candidate non-Abelian states. This task is harder than obtaining evidence for non-Abelian topological order. It relies on counting both fractional and integer contributions to the system's thermal conductance, which, in turn, requires certain assumptions to be made about the process by which different propagating modes reach thermal equilibrium $^{10}$. The issue of equilibration is further complicated by the fact that the edge modes can reach equilibrium not only with each other, but also with lattice vibrations called phonons, which provide an unwanted contribution to the thermal conductance.

Banerjee et al. went to great lengths to minimize this phonon contribution. They carried out their experiments at temperatures of about $20 \mathrm{mK}$ and used a sophisticated design of a source and drains to avoid the coupling of edge modes to phonons. By comparison, Kasahara and colleagues' experiment was much less intricate and required temperatures of only about $5 \mathrm{~K}$. These authors could not detect a signal of half-integer quantization at lower temperatures, which probably suggests that the system transitioned to a different phase. Their results also indicate that a substantial amount of heat was carried by phonons.

Under these circumstances, it should be surprising that the authors saw signs of quantized Hall heat transport - the heat conduction in the direction perpendicular to that of the thermal gradient - by Majorana fermions. However, two recent studies ${ }^{11,12}$ have argued that phonon coupling not only is not detrimental, but also can actually be necessary for the observation of such an effect. More work, both theoretical and experimental, is required to fully understand the implications of these experiments. Nevertheless, it is undoubtedly exciting that the quest for Majorana fermions is heating up in this manner.

Kirill Shtengel is in the Department of Physics and Astronomy, University of California, Riverside, California 92521, USA. e-mail:kirill.shtengel@ucr.edu

1. Majorana, E. Nuovo Cimento 14, 171-184 (1937)

2. Wilczek, F. Nature Phys. 5, 614-618 (2009)

3. Banerjee, M. et al. Nature 559, 205-210 (2018).

4. Kasahara, Y. et al. Nature 559, 227-231 (2018).

5. Read, N. \& Green, D. Phys. Rev. B 61, 10267-10297 (2000).

6. Kitaev, A. Ann. Phys. 321, 2-111 (2006).

7. Jackeli, G. \& Khaliullin, G. Phys. Rev. Lett. 102 017205 (2009).

8. Mourik, V. et al. Science 336, 1003-1007 (2012).

9. He, Q. L. et al. Science 357, 294-299 (2017).

10.Simon S. H. Phys. Rev. B 97, 121406 (2018).

11.Ye, M., Halász, G. B., Savary, L. \& Balents, L. Preprint at https://arxiv.org/abs/1805.10532 (2018).

12.Vinkler-Aviv, Y. \& Rosch, A. Preprint at https://arxiv. org/abs/1805.11587 (2018).

\title{
How rats wreak havoc on coral reefs
}

\begin{abstract}
The introduction of non-native rats can devastate island ecosystems. It now emerges that these rats also harm a complex web of interactions linking seabirds with the algae and fishes of nearby coral reefs. SEE LETTER P250
\end{abstract}

\section{NANCY KNOWLTON}

$\mathrm{N}$ on-native rats that invade tropical islands can cause problems for the ecosystems they invade ${ }^{1}$. These intruders can decimate the native populations on which they feed, such as plants and terrestrial invertebrates. Bird populations can plummet, too, when rats eat eggs and nestlings. The complex, indirect effects of rodent presence can spread deeply and widely through island food webs ${ }^{2}$. However, little attention has been paid to the indirect impacts of such invasive species on adjacent coral-reef communities. On page 250, Graham et al. ${ }^{3}$ address this for sites on the Chagos Archipelago in the Indian Ocean (Fig. 1), comparing coral reefs surrounding six rat-infested islands with those adjacent to six islands that lacked rats. The authors find that reefs near rat-infested islands have fewer nutrients, fewer fishes and reduced numbers of fishes grazing on the algae that compete with corals.

One of the most marked effects of rats was a 760 -fold decline in the number of nesting seabirds per hectare on rat-infested islands compared with rat-free islands. On the latter islands, the larger populations of birds produced larger deposits of guano - nitrogenrich bird excrement. This nitrogen is mostly obtained from food that the birds consume during long-distance foraging trips to parts of the ocean that, thanks to their higher levels of nutrients, are 100 to 100,000 times more productive than the waters in the immediate vicinity of an island. The nitrogen deposition rates on the rat-free islands were 251 times greater per hectare than were those on the rat-infested islands. Using a technique to identify different isotopic forms of nitrogen, the authors could distinguish this 'imported' seabird nitrogen from locally derived nitrogen. This enabled Graham and colleagues to track where the seabird-deposited nitrogen ended up.

Some nitrogen was absorbed by plants on the islands, and some entered the ocean through rain or breaking waves. For example, 100 metres from the shore of rat-free islands, both a type of sponge and a type of macroalga had elevated levels of nitrogen derived from seabird foraging, compared with the levels recorded near rat-infested islands. At 230 metres from the shore of rat-free islands, the concentration of seabird-derived nitrogen 
$\sum$ in turf algae and in the muscle tissue of a species of alga-eating damselfish was higher than such measurements on rat-infested islands. By measuring growth rings in damselfish ear bones, Graham and colleagues showed that the damselfish in the waters around rat-free islands grew faster and at any given age were larger than those living beside rat-infested islands, presumably because their food was richer in nitrogen.

Looking at all types of reef fish, the authors found that the total biomass of the fish population was $48 \%$ higher around rat-free islands than around rat-infested islands. Moreover, of all the types of reef fish, the abundance of herbivorous (alga-eating) fishes was the most negatively affected by the presence of rats. Herbivorous fish are particularly important for coral reefs, because their grazing prevents the algae from overgrowing and killing corals. Around rat-free islands, parrotfishes, a group of herbivores, grazed the entire surface of the reef 9 times per year, whereas around rat-infested islands the equivalent figure was only 2.8 times per year. Because parrotfishes feed with powerful beaks, there was also more bioerosion and greater production of sand on coral reefs surrounding rat-free islands; however, the amount of living coral was not lower than that on rat-infested islands.

The dramatic effects that Graham and colleagues document provide a comprehensive picture of how reefs are interconnected with the surrounding land and seascapes. The movement of organisms around such habitats generates genetic connections between regions that have long been appreciated by biogeographers and geneticists. In marine conservation, the generation of networks of marine-protected areas that take into account this genetic connectivity between coral reefs is increasingly a part of the planning process. John Donne's poem 'No Man is an Island' explores the nature of human connections, and, as others have noted ${ }^{4}$ in a similar vein, "no reef is an island", either.

However, the nutritional (trophic) connections linking coral reefs with other marine and terrestrial ecosystems haven't been studied as extensively as have the genetic connections. This is surprising, given the long-standing puzzle of how coral reefs thrive in nutrient-poor waters - a phenomenon commonly called Darwin's paradox because Charles Darwin highlighted this enigma. Tight recycling of energy and nutrients certainly helps ${ }^{5}$, as do the oceanic plankton that are captured by the proverbial "wall of mouths" that the fishes on a reef present ${ }^{6}$.

Graham and colleagues' study adds to our growing appreciation of the importance of long-distance nutritional subsidies for reefs, generated not only by seabirds as documented here, but also by wide-ranging underwater predators such as sharks ${ }^{7}$. Notably, human impacts can disrupt the subsidies in both of these cases.

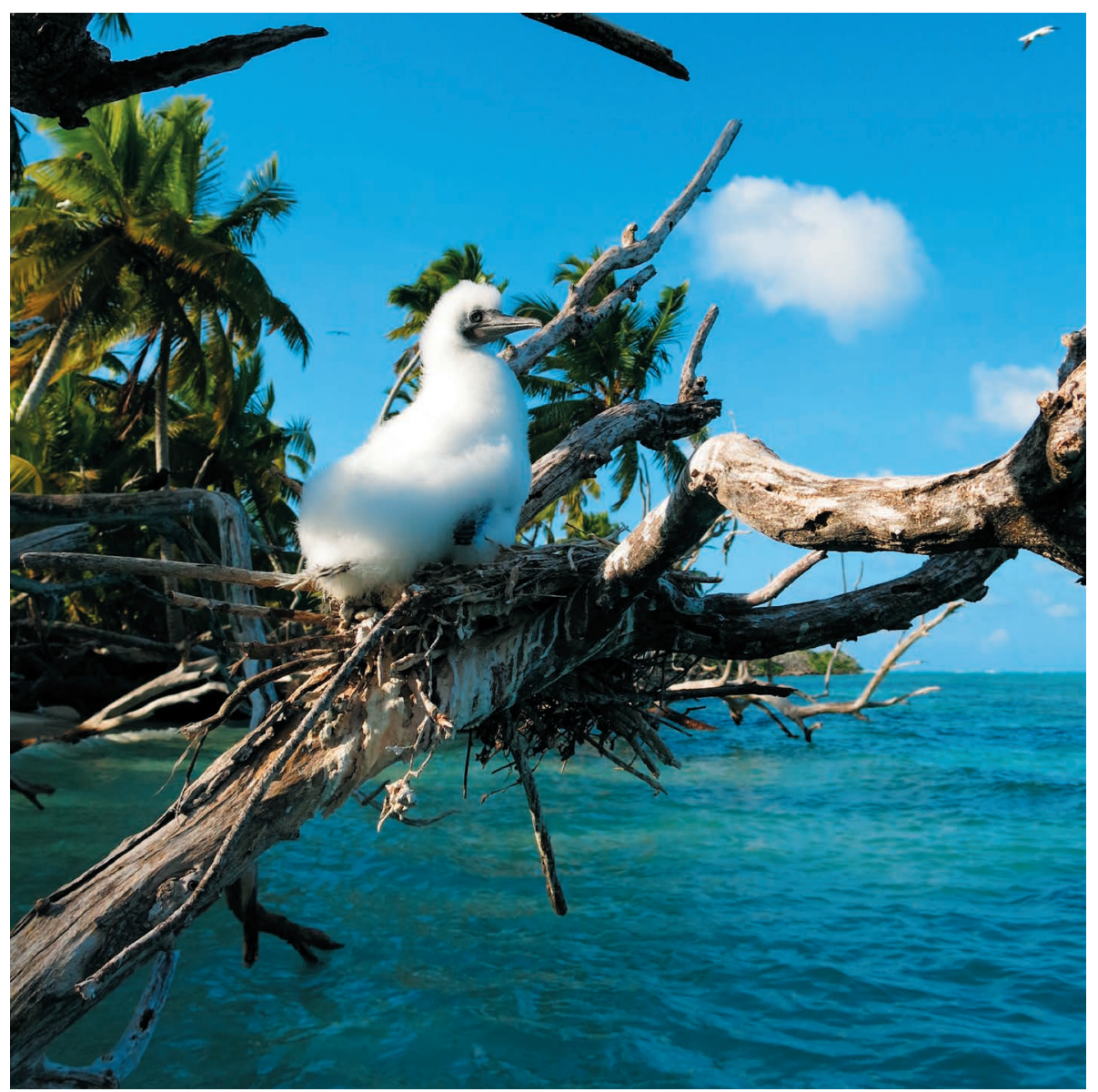

Figure 1 | Booby chick on a nest above a coral-reef lagoon in the Chagos Archipelago. Graham et al. report their studies of how non-native rats affect the ecosystems of islands and adjacent coral reefs in the Chagos Archipelago in the Indian Ocean. They find that rat-free islands have substantially more seabirds than do rat-infested islands. Moreover, nitrogen deposits from seabird excrement has a positive effect on nearby coral reefs through nutrient cycling, which generates nitrogen-rich algae that boost the fish population.

This work has immediate practical implications, particularly because reefs are under grave threat around the world. Many of the early losses of coral reefs were due to overfishing $^{8}$, and a scarcity of herbivorous fishes continues to make reefs less resilient ${ }^{9,10}$. Now, however, one of the major causes for concern over reef survival is the impact of climate change and the ability of reefs to recover from disturbances due to oceanic warming, which is causing mass coral bleaching and death affecting even remote and protected reefs ${ }^{11}$.

Adding rats to the list of dangers to reefs might seem discouraging. Yet the discovery of the negative impacts of rats on reefs does point directly to a specific strategy that could slow the pace of reef degradation. Rats and other invasive mammals have been successfully eradicated from hundreds of islands ${ }^{12}$, with beneficial effects on many terrestrial ecosystems. Graham and colleagues suggest that the same strategy, and others more generally aimed at protecting seabirds, should be a priority for islands associated with coral reefs, helping to buy time while society comes to grips with and, one must hope, slows climate change. In the meantime, scientists will now be on the lookout for how these rat-infested versus rat-free islands recover from the surely inevitable next coral-bleaching event.

Nancy Knowlton is in the Department of Invertebrate Zoology, National Museum of Natural History, Smithsonian Institution, Washington DC 20013, USA.

e-mail:knowlton@si.edu

1. Harper, G. A. \& Bunbury, N. Glob. Ecol. Conserv. 3 607-627 (2015).

2. Nigro, K. M. et al. Restor. Ecol. 25, 1015-1025 (2017).

3. Graham, N. A. J. et al. Nature 559, 250-253 (2018),

4. Schill, S. R. et al. PLoS ONE 10, e0144199 (2015).

5. de Goeij, J. M. et al. Science 342, 108-110 (2013).

6. Hamner, W. M., Colin, P. L. \& Hamner, P. P. Mar. Ecol. Prog. Ser. 334, 83-92 (2007).

7. Williams, J. J., Papastamatiou, Y. P., Caselle, J. E., Bradley, D. \& Jacoby, D. M. P. Proc. R. Soc. B $\mathbf{2 8 5}$, 20172456 (2018).

8. Pandolfi, J. M. et al. Science 301, 955-958 (2003).

9. Jackson, J. B. C., Donovan, M. K., Cramer, K. L. \& Lam, V. (eds) Status and Trends of Caribbean Coral Reefs: 1970-2012 (Glob. Coral Reef Monit. Netw. IUCN, 2014); go.nature.com/2jdpbvp

10.Adam, T. C., Burkepile, D. E., Ruttenberg, B. I. \& Paddack, M. J. Mar. Ecol. Prog. Ser. 520, 1-20 (2015).

11. Hughes, T. P. et al. Nature 556, 492-496 (2018).

12.Russell, J. C. \& Holmes, N. D. Biol. Conserv. 185, 1-7 (2015). 